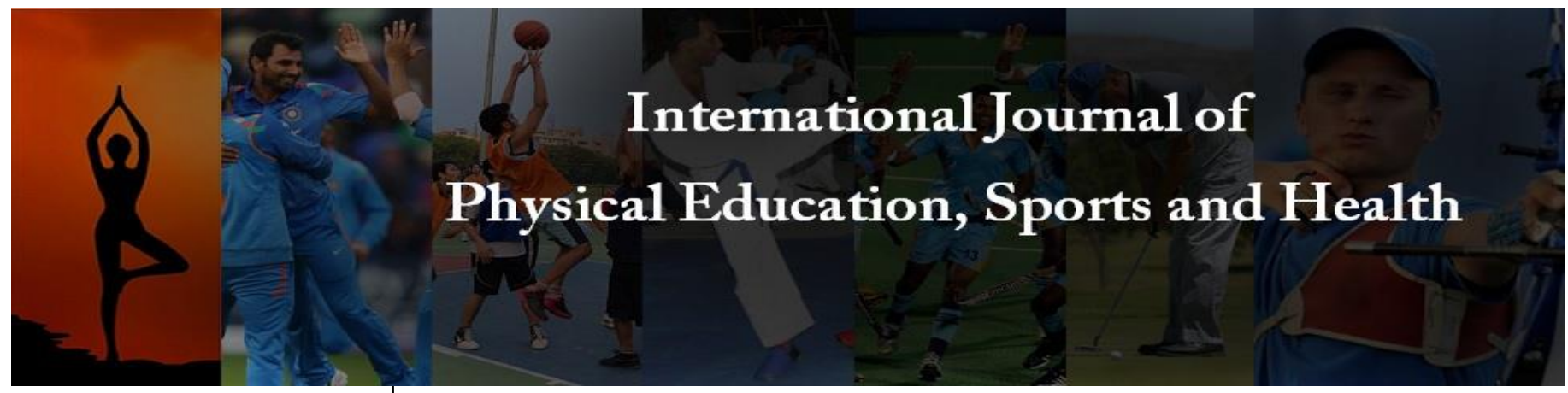

P-ISSN: 2394-1685

E-ISSN: 2394-1693

Impact Factor (ISRA): 5.38

IJPESH 2021; 8(3): 275-279

(C) 2021 IJPESH

www.kheljournal.com

Received: 04-03-2021

Accepted: 06-04-2021

\section{Xiang Qian}

Asst. PhD, Department of

Combat Sports and Power

Sports, Faculty of Sports and

Management, National

University of Physical Education

and Sport of Ukraine, Ukraine

\section{G Korobeynikov}

Professor, Ph.D., Department

Director, Department of Combat

Sports and Power Sports,

Faculty of Sports and

Management, National

University of Physical Edu

Cation and Sport of Ukraine,

Street Physical Culture, 1, Kyiv,

Ukraine

L Korobeynikova

Professor, Ph.D., Department of

Psychology and Pedagogy,

Faculty of Health, Physical

Education and Tourism,

National University of Physical

Education and Sport of Ukraine, Street Physical Culture, 1, Kyiv,

Ukraine
Corresponding Author:

G Korobeynikov

Professor, Ph.D., Department

Director, Department of Combat

Sports and Power Sports,

Faculty of Sports and

Management, National

University of Physical Edu

Cation and Sport of Ukraine,

Street Physical Culture, 1, Kyiv,

Ukraine

\section{Analysis of athletes competition videos based on hypothesis testing: Case study of elite badminton single players}

\section{Xiang Qian, G Korobeynikov and L Korobeynikova}

DOI: https://doi.org/10.22271/kheljournal.2021.v8.i3e.2095

\begin{abstract}
The study aimed to establish a general framework for the analysis of the athletes' competition videos through two badminton games ( $\mathrm{n}=5$ sets) between Kento Momota and Viktor Axelsen. Version 3.8 of the data analysis software Python was used. The results of the study showed that for Momota and Axelsen, the percentages of winning long rallies were 52.05 and 46.48 , respectively and the percentages of winning in monster rallies were 62.50 and 57.14, respectively. When the number of shots in a rally was greater than 14.40, the rallies won by Momota and Axelsen were 7.60 and 6.60, respectively. And winner of the subsequent rally after 14.40 , the rallies won by the two were 9.40 rallies and 4.40 rallies. When Momota hit less diagonally in the subsequent rally, he won 58 of 90 rallies, with a success rate of $64.44 \%$. Therefore, the three hypotheses of two variables and their quantitative research resulted in the hypothesis of a significance level of $\mathrm{p} \leq 0.01$, respectively. the theoretical contribution of this article is to put forward a research on the video of the athlete's game and construct an effective theoretical model, which provide a reference for the coaching team and the athletes.
\end{abstract}

Keywords: elite players, case study, badminton match, hypothetical testing

\section{Introduction}

Badminton is a racquet sport characterised by high-intensity ${ }^{[1,2]}$, intermittent actions ${ }^{[3-5]}$. Since badminton is a non-contact game, where players from the opposing teams are separated by the net, the techniques and tactics involved as well as the flexible usage during the competitions result in a non-linear relationship between badminton competition result and sports quality. It is difficult for the coaches and others to predict an athlete's competition results based on his/her sports quality. Therefore, match statistical analysis of the badminton video is vital to show details of the frequency of skills and tactics of each player. The use of this knowledge further enables the coaches to provide feedback to the players on the playing pattern, and physiological and psychological stress ${ }^{[6]}$ of the performers.

With the continuous improvement of badminton players' competitive level, scientific and perfect training methods and efficient and intelligent technical and tactical analysis and decision-making mechanisms after the game are increasingly required to improve the badminton players' competitive level to meet the changing structure of the game. Especially in elite badminton players, the quantitative analysis of variables related to time ${ }^{[7,8]}$ and skills and tactics in the game ${ }^{[9]}$ has attracted great attention from related researchers ${ }^{[10,5]}$. In addition, the above-mentioned researchers also conducted a descriptive and quantitative analysis of the badminton game video to study the specific performance of badminton players in the game ${ }^{[4]}$. Other scholars have also used intelligent information processing technology to provide crossdisciplinary research on the anthropometry of badminton players and the biomechanics of specific movements and shots. With the technological advancement of sports science, image recognition technology, data collection, and computer action recognition and analysis have become more and more common in the scientific research of badminton.

Prior research had pointed out more specific quantitative analysis and hybrid methods for nonparametric data of badminton video analysis. 
However, we argue that while quantitative analysis of badminton video can generate positive feedback for athletes and coaches to a certain extent, the effectiveness and repeatability of video analysis methods are yet to be tested. Followed by the analysis of video recordings of competitions lacks the support of a theoretical framework. In addition, the validity and repeatability of statistical variable indicators by athletes, and the impact on athletes are yet to be tested. Against the abovementioned background, this study the videos of two matches ( $\mathrm{n}=5$ sets) between Japanese badminton player Momota and Danish badminton player Axelsen, who are the most representative players of the World Badminton Federation currently, are taken as the case study material to investigate data of statistical variables, put forward the hypotheses of this research and test the hypotheses.

\section{Theoretical Framework and Hypothesis \\ 2.1 Procedures}

The empirical analysis of the videos of the competition between elite badminton players Momota and Axelsen helps coaches and researchers to grasp the technical and tactical characteristics of athletes and opponents in a more intuitive and effective manner, thereby improving the pertinence and effectiveness of the pre-competition training plan. Combining with the purpose of this article, we have constructed a theoretical model for the analysis of videos of elite badminton players' competitions, including the following five basic steps.

\subsubsection{Collecting Data}

According to the current rules of the Badminton World Federation

(http://www.internationalbadminton.org/statues.asp), two badminton matches ( $\mathrm{n}=5$ sets) played between Momota (Age: 26, Height: $175.00 \mathrm{~cm}$ ) and Axelsen (Age: 27, Height 194.00 $\mathrm{cm})$ were randomly drawn as the test samples. The video platform YouTube was used for the statistical analysis of video of the badminton matches. The playback speed of the video is an important factor in analysing the outcomes of the techniques and tactics used in badminton matches. As per Zhang et al. ${ }^{[11]}$ for the analysis of the techniques and tactics used in badminton matches, the ideal playback speed of the video is $75 \%$. The samples were collected from the 2019 and 2020 World Badminton Super Series. All cases of badminton videos were downloaded from YouTube (https://www.youtube.com/watch?v=boQC4J4E1ZQ\&\&t=96 6s;https://www.youtube.com/watch?v=N99YOz_DaJ0\&\&t=5 023s).

\subsubsection{Performing Data Reliability Test}

First, to ensure the validity of the collected data and avoid artificial errors, two observers (Professors having the qualification required by the National University of Ukraine on Physical Education and Sport) observed the same videos independently and obtained two sets of observed data. Second, hypotheses were developed that there are no significant differences and there are significant differences between the two sets of data. Third, a two-sample t test was performed on the two sets of data collected by the observers. By performing the two-sample t-test on the proposed hypotheses, it was found that the hypothesis is a two-sample test of mean (two-sided test). The sample size is $\mathbf{n}_{1}=\mathbf{n}_{2}=7$, which is a small sample. Therefore, the t-test statistic (generally, $n \geq 30$ is considered to be a large

sample) $T=\frac{(\bar{X}-\bar{Y})-\left(\mu_{1}-\mu_{2}\right)}{S_{w} \sqrt{\frac{1}{n_{1}}+\frac{1}{n_{2}}}}$ is used, among which the

test statistic

$$
S_{w}=\frac{\left(n_{1}-1\right) s_{1}^{2}+\left(n_{2}-1\right) s_{2}^{2}}{n_{1}+n_{2}-2}
$$
follows the

$t_{\text {distribution with the parameter }} n_{1}+n_{2}-2$, and the calculation results of the data is $|\boldsymbol{T}|=\mathbf{0 . 0 1}$

Obviously, $\quad|\boldsymbol{T}|=\mathbf{0 . 0 1}<0.70$, therefore, the null hypothesis is not rejected, that is, there is no significant differences between the two sets of data. Accordingly, it can be considered that: There is no significant differences between the two independent sets of data collected by two observers respectively, indicating that the error of manual observation is very small and the data are completely reliable.

\subsubsection{Formulating Hypotheses}

There is no fixed and unified standard for establishing the hypotheses of the propositions H0 (null hypothesis) and H1 (alternative hypothesis) ${ }^{[12]}$, and the determination of the hypothesis depends on the problem to be tested and the purpose of the test. The proposition which is intended to be proven is formulated in the alternative hypothesis, while the original, traditional viewpoint or conclusion is formulated in the null hypothesis. Therefore, the hypothesis of this research is based on the empirical analysis of the video of badminton matches between the elites. Based on the proposition that Momota makes better use of rallies and diagonals than Axelsen, the following hypotheses were proposed: H0: $\mu_{1} \geq \mu_{2, \mathrm{H} 1:} \mu_{1}<\mu_{2}$.

\subsubsection{Analysing Data to Test the Hypotheses}

Hypothesis testing ${ }^{[13]}$ is a systematic way to select samples from a group or population with the intent of determining the expected behaviour of the entire group. Hypothesis testing can be divided into: t-test, z-test, and f-test. The t-test uses a small sample to test the overall parameters. The characteristic of the test is that it can test the significance of the sample mean when the mean squared error is unknown. It is of two types: one-sided test and two-sided test. The sample size of the collected videos of badminton matches in this study is small $(\mathrm{n}<30)$. Since the question is a two-sample test of mean (one-sided test), and the sample size, $n_{1}=n_{2}=5$ is small ( $n \geq 30$ is considered a large sample), t-test was used to test the hypothesis.

\subsubsection{Drawing Conclusions}

We determined the critical point $\mathrm{C}$ of the distribution and the test rules, brought in the formula to calculate the value of the test statistic, compared the conclusions drawn and made the test judgments.

\subsection{Theoretical Hypothesis}

An effective theoretical model of technical and tactical analysis is of great significance for promoting the performance level of athletes. Base on the abovementioned five steps of the theoretical model designed for the case study of elite badminton players, statistical analysis is first 
conducted on the videos of the matches between Momota and Axelsen. Rally and Diagonal are set as the variable indicators while the theoretical hypotheses of this research are put forward.

\section{This leads to the following hypotheses}

H1: Momota hits the diagonal line less than the opponent, and his diagonal scoring rate is higher than the opponent, i.e., H0 a: $\mu_{1} \geq \mu_{2}$, H1 a: $\mu_{1}<\mu_{2}$.

$\mathrm{H} 2$ : Momota is better at scoring in long rallies, and exceptionally better than the opponent in monster rallies, i.e., H0 b: $\mu_{1} \geq \mu_{2}$, H1 b: $\mu_{1}<\mu_{2}$

H3: In the subsequent sets after the opponent uses the diagonal lines for more times, Momota scores more than the opponent in the rallies with diagonal, i.e., $\mathrm{H} 0 \mathrm{c}$ : $\mu_{1} \geq \mu_{2}$, H1 c: $\mu_{1}<\mu_{2}$.

\section{Methods}

\subsection{Variable Definitions}

Rally ${ }^{[14]}$ refers to a single play between the two sides. The concept of rally is widely used in sports data analysis, such as racquet sports, basketball, and volleyball. In volleyball games, rally is defined ${ }^{[15]}$ as: "The ball passed over the net once, to prove that the two sides had competed for one time. This is defined as a rally". Based on previous studies [16, 7, 17], we define rally in badminton as follows: Using the technique of overhand stroke, a badminton player hits the shuttle, causing the shuttle to pass over the net once, to prove that the two sides had competed for one time. Additionally, for a more intuitive understanding of the number of hits and the percentage of points scored of the athletes per competition and per set, four lengths of a rally were determined in this study, based on the lengths of the rallies and the number of rallies, as shown in Table 1.

Table 1: The statistics of the length of rallies and rally shots of Kento Momota and Victor Axelsen for two badminton matches (five sets)

\begin{tabular}{|c|c|c|c|}
\hline Length of a Rally & Number & Kento Momota & Viktor Axelsen \\
\hline $0-10$ & 85 & 50 & 35 \\
\hline $11-20$ & 50 & 24 & 26 \\
\hline $21-30$ & 31 & 18 & 13 \\
\hline $31+$ & 16 & 10 & 6 \\
\hline Sum & 182 & 102 & 80 \\
\hline
\end{tabular}

The definition of the concept of diagonal in the literature is "the line segment combining nonadjacent corners of a polygon" [18]. Based on the definition previously proposed by other scholars ${ }^{[19]}$, this study defined the diagonal lob from the backcourt of badminton as follows-from the vertex of his/her own service court, the player hits the shuttle and cause it to pass over to the diagonal court of the opponent along a certain curve. It includes diagonal clear, diagonal attack clear and diagonal drive. The badminton court has a length of $13.40 \mathrm{~m}$, singles width $5.18 \mathrm{~m}$, singles stadium diagonal length = $14.366 \mathrm{~m}$

\subsection{Data Analysis}

The hypothesis testing method was used in this study to analyse the statistical results of the videos of the elite badminton matches. Version 3.8 of the data analysis software Python was used.

\subsection{Hypothesis Verification}

For the hypotheses proposed based on the video analysis of elite badminton players, we found that the question is a twosample test of mean (one-sided test). The sample size is $n_{1}=n_{2}=5$, which

is a small sample. Therefore, the t-test statistic was used: $T=\frac{(\bar{X}-\bar{Y})-\left(\mu_{1}-\mu_{2}\right)}{S_{w} \sqrt{\frac{1}{n_{1}}+\frac{1}{n_{2}}}}$ , while the t-test statistic follows the $n_{1}+n_{2}-2$ distribution with $t$ parameter, and the verification results of the null hypothesis and the alternative hypothesis are calculated, as shown in Table 2.

Table 2: Hypothesis Testing Results

\begin{tabular}{|c|c|c|}
\hline Hypothesis & T-stat & Results \\
\hline $\begin{array}{c}\text { Hypothesis 1: Kento Momota hits the } \\
\text { diagonal line less than the opponent, and } \\
\text { his diagonal scoring rate is higher than the } \\
\text { opponent. }\end{array}$ & $3.38(* * *)$ & Supportive \\
\hline $\begin{array}{c}\text { Hypothesis 2: Kento Momota is better at } \\
\text { scoring in long rallies, and exceptionally } \\
\text { better than the opponent in monster } \\
\text { rallies. }\end{array}$ & $5.48(* * *)$ & Supportive \\
\hline $\begin{array}{c}\text { Hypothesis 3: In the subsequent sets after } \\
\text { the opponent uses the diagonal lines more } \\
\text { times, Kento Momota scores more than } \\
\text { the opponent in the rallies with diagonal. }\end{array}$ & $2.90(* * *)$ & Supportive \\
\hline Note. $* \mathrm{p}<0.1 ; * * \mathrm{p}<0.05 ; * * * \mathrm{p}<0.01$ & & \\
\hline
\end{tabular}

\section{Results and Discussion}

\subsection{Results}

The hypothesis test result (Table 2) clearly shows in a more comprehensible format that $\mathrm{H} 1$ shows a t-stat of 3.38 and supports the hypothesis. $\mathrm{H} 2$ also shows a t-stat of 5.48 supporting the hypothesis. H3 results state a t-stat of 2.90 . This also supports the hypothesis.

Hypothesis 1(H1): The test result found that Momota hits the diagonal line less than the opponent, and his diagonal scoring rate is higher than that of the opponent, a fact validated by the hypothesis significance $\mathrm{p} \leq 0.01$.

Hypothesis $2(\mathrm{H} 2)$ : The test result found that Momota is better at scoring in long rallies, and performs better than the opponent in long rallies with more hits, a fact validated by the hypothesis significance $\mathrm{p} \leq 0.01$.

Hypothesis 3 (H3): The test result found that after the opponent uses the diagonal lines more times, Momota has a high scoring rate in the subsequent rally with diagonal and in the second or third set, a fact validated by the hypothesis significance $\mathrm{p} \leq 0.01$.

\subsection{Discussion}

Analysis of the rally variables. The establishment of the concept of rally is a further subdivision and dynamic description of the competition process. Each point is generated when both sides of the competitions hit the shuttle back and forth over the net until a player makes the bird dead. Therefore, through the analysis of the organisational structure of the rally system, the modes and distributions of the points won and lost in different rallies, as well as the mutual influence and interaction between different rallies are found to understand the laws of winning of elite badminton players. In addition, not only can rally variables solve the problem in comparing data indicators between different players, they also 
can effectively solve the issues of comparing the data between different sample sizes. The standardisation based on the concept of "rally" also reflects a basic way of thinking in data analysis. Therefore, the data analysis of badminton urgently needs to be standardised based on rally.

In the finals of All English Open Championships 2019 and Perodua Malaisia Master 2020, the shots were 2620, the rallies were 182, the average shots per rally (AVG shots) was 14.40, long rallies 71 , accounting for $39.01 \%$. For Momota and Axelsen, the percentage of winning long rallies were 52.05 and 46.48, respectively. Monster rallies (> 30 shots) were 16 , accounting for $8.79 \%$. For Momota and Axelsen, the percentage of winning monster rallies were 62.50 and 57.14, respectively. Moreover, if the number of shots rally was greater than 14.40, the rallies won by Momota and Axelsen were 7.60 and 6.60, respectively. Winner of the next after rally of 14.40 shots, the rallies won by Momota and Axelsen were 9.40 and 4.40 , respectively. The average shots per rally in the abovementioned five sets of games between the elite badminton players was 14.40 , which is consistent with the research of scholars in relevant fields ${ }^{[5,20]}$. It is evident from Table 1 that Momota and Axelsen used most frequently in the rallies in the range of $0-10$ and 11-20, while in the rallies in the range of 21-30 and 30+, Momota was better than Axelsen in attack efficiency and defensive efficiency. However, in the second set of games in the All English Open Championships finals, among three of the four ranges of the rallies, Momota's scores were lower than Axelsen, and the game entered the tiebreaker. Therefore, we found that Momota is better at scoring in long rallies during the game, and exceptionally better than the opponent in monster rallies with more hits, which is consistent with Hypothesis 2. Compared with Axelsen, we suggest that in long rallies and in monster rallies, the defensive efficiency should be strengthened to enhance scoring ability.

To sum up, the division of rallies are not only helpful for the athletes to check and understand the attack efficiency and defensive efficiency exposed by themselves or their opponents during the rallies, but it also helps them to "strengthen the scoring ability in rallies and make up for their shortcomings" with pertinence. The scoring ability of each round (rally length) can make up for the shortcomings. It can also be used as an indicator for the reference of young athletes in multi-ball practice.

Diagonal variable. Diagonal lobs from the backcourt fly the longest running line on the badminton court. The tactical intent is to disrupt the opponent's moving rhythm and pace on the field by surprise to get oneself out of the passive situation caused by the previous hit in the rally. Diagonal lobs from the backcourt of badminton consist of three key elements. The first is the height, the second is the spatial position of the backcourt, and the third is the diagonal line. There is no strict definition of the "height" of a diagonal lob from the backcourt of badminton, but the difference in height reflects the attributes of attack and defence. "Diagonal line" refers to the direction of the lob. In the game, intentional hits on the diagonal line by the athletes are out of specific tactical purposes. The shots of high-quality diagonal lobs are constrained by the long flying line along the diagonal line, as well as the speed and the altitude of the shuttle. Of course, in the game, if an athlete shoots a diagonal lob from the backcourt in a reasonable way, it poses the greatest threat to the opponent.

It is evident from the statistical analysis of the diagonal lobs from the backcourt in the competitions between Momota and
Axelsen that, in the finals of All English Open Championships 2019 and Perodua Malaisia Master 2020 finals, the diagonals hit by Momota and Axelsen were 268 shots and 380 shots, accounting for $10.23 \%$ and $14.50 \%$, of the total shots, respectively. Analysis of the above data proves that Axelsen used the diagonal $70.53 \%$ more than the opponent. The average percentage of diagonals per rally is 1.47 and 2.08, respectively. When Axelsen used the diagonal lobs, his main intention was to affect Momota's position on the court, and look for the loopholes in the shots (or diagonals that are not in place) of the opponent to create opportunities for himself to attack and press down. However, the above data do not reflect the effectiveness of the diagonals used by Axelsen. As we explore Momota and Axelsen's performance in this game, Momota has 182 rallies with 42 diagonals, the percentage is 23.08, and Axelsen has 182 rallies with 94 diagonals, the percentage is 51.65. In the whole game, Momota won 24 out of 42 rallies with diagonals and the efficiency value of scoring with diagonals was as high as $57.14 \%$. While Axelsen won 42 out of 94 rallies with diagonals, the efficiency value of scoring with diagonals was only $44.68 \%$. This is consistent with Hypothesis 1. In addition, when Momota had fewer counterattacks on the diagonal, he won 58 of 90 rallies subsequently, with a success rate of $64.44 \%$. This is consistent with Hypothesis 3 in this study. Therefore, through the analysis of the diagonal lops hit by Momota and Axelsen, we found that although Axelsen increased the opponent's running range on the badminton court through the diagonal line, while he continued to consume the opponent's physical energy, his own physical energy would be under more pressure. Therefore, for elite badminton singles players, the use of diagonal techniques and tactics in the game has to fit the different needs of players.

\section{Conclusion}

Variable is an effective quantitative analysis tool for the statistics and analysis of game videos. However, any single variable can only explain the effects and influences when used by the athletes in the game. Each variable has its limitations, the direct impact of the variables' indicators on the outcome of the game should not be considered in an isolated manner. Rather, variables should be used to support the theory of this research. This research conducts a case study based on the videos of badminton game between Momota and Axelsen. Through the analysis of the actual data collected, the proposed theoretical model was verified. Although there are problems such as insufficient statistical sample size, and the hypothesis testing methods are borrowed from existing research, for the new questions we want to investigate, the problems do not pose any objective influence on the results of the research. In the follow-up research, especially in the case studies of athletes, issues including the magnitude of the statistical sample size, and selection of sample types, such as the winning and loss of the athletes in the games, need to be further improved. Based on badminton video footages, this study selected elite badminton players Momota and Axelsen for empirical research, used rally and diagonal lobs as variable indicators to perform high-level statistics on the video of matches, analysed the use of variable indicators for the proposal of the hypotheses of the question, and employed hypothesis testing methods to verify the validity and repeatability of the analysis of the video footages of elite athletes based on the variable indicators. Additionally, the theoretical contribution of this article is to put forward a technical and tactical research on the video of the athlete's 
game and construct an effective theoretical model, which provide a reference for the coaching team and the athletes, and help them to design and formulate skills and tactics before important competitions. It can be used as a model for coaches and athletes to analyse the opponents, and has practical value in further evaluating athletes, evaluating the technical and tactical characteristics of their own athletes and opponents, and revealing tactics and strategies of athletes.

\section{Acknowledgments}

Xiang-Qian Xu has conceived and designed the study. XiangQian $\mathrm{Xu}$ has analysed the data. Xiang-Qian Xu drafted the manuscript. Georgiy Korobeynikov and Lesya Korobeynikova advised on analysis and interpretation of the data and critically revised the manuscript.

\section{References}

1. Alcock A, Cable NT. A comparison of singles and doubles badminton: heart rate response, player profiles and game characteristics. International Journal of Performance Analysis in Sport 2009;9(2):228-237.

2. Abián-Vicén J, Del Coso J, González-Millán C, Salinero JJ, Abián P. Analysis of dehydration and strength in elite badminton players. PloS one 2012;7(5):e37821.

3. Faccini P, Dal Monte A. Physiologic demands of badminton match play. The American journal of sports medicine 1996;24(6_suppl):S64-S66.

4. Phomsoupha M, Laffaye G. The science of badminton: game characteristics, anthropometry, physiology, visual fitness and biomechanics. Sports medicine 2015;45(4):473-495.

5. Gomez MÁ, Rivas F, Connor JD, Leicht AS. Performance differences of temporal parameters and point outcome between elite men's and women's badminton players according to match-related contexts. International journal of environmental research and public health 2019;16(21):4057.

6. Josue F, Abdullah MF, Zulkapri I, Soeed K, Tariq I. Movement pattern in term of court coverage among top international male and female badminton players during BWF World Championships 2013. Jurnal Sains Sukan \& Pendidikan Jasmani 2020;9(1):9-14.

7. Abian-Vicen J, Castanedo A, Abian P, Sampedro J. Temporal and notational comparison of badminton matches between men's singles and women's singles. Int. J. Perform. Anal. Sport 2013;13:310-320.

8. Gawin W, Beyer C, Seidler M. A competition analysis of the single and double disciplines in world-class badminton. Int. J. Perform. Anal. Sport 2015;15:9971006.

9. Aydogmus M, Arslanoglu E, Senel O. Analysis of badminton competitions in 2012 London Olympics. Turkish Journal of Sport and Exercise 2014;16(3):55-60.

10. Torres-Luque G, Fernández-García ÁI, Blanca-Torres JC, Kondric M, Cabello-Manrique D. Statistical Differences in Set Analysis in Badminton at the RIO 2016 Olympic Games. Frontiers in Psychology 2019;10:731-731.

11. Zhang H, Li XD, FY. Application of computer video technique to analysis of table tennis matches. Journal Shanghai University of Sport 2005;29(6):47-50.

12. Poincaré H. Science and hypothesis. Science Press 1905.

13. Raftery AE, Gilks WR, Richardson S, Spiegelhalter D. Hypothesis testing and model. Markov chain Monte Carlo in practice 1996, 163-185.
14. Lu S, Ding S. Modern Chinese dictionary. Beijing: The Commercial Press 1989, 679.

15. Weng B. Research on the characteristics of the rounds between the Chinese Women's Volleyball Team and the World Women's Volleyball Team. Journal of Xi'an Institute of Physical Education 2000;17(04):37-39.

16. Faude O, Meyer T, Rosenberger F, Fries M, Huber G, Kindermann W. Physiological characteristics of badminton match play. European journal of applied physiology 2007;100(4):479-485.

17. Courel-Ibáñez J, Martínez BJSA, Cañas J. Game performance and length of rally in professional padel players. Journal of Human Kinetics 2017;55(1):161-169.

18. Çoker D, Karaçay T. Matematik terimleri sözlüğü. Türk Dil Kurumu Yayınları 1983.

19. Zhang B, Liu Y, Dou H. Badminton. Beijing Book Co., Ltd 2017.

20. Gomez MA, Leicht AS, Rivas F, Furley P. Long rallies and next rally performances in elite men's and women's badminton. PloS one 2020;15(3):e0229604. 